\title{
El cambio climático, ¿reto para la Responsabilidad Social Empresarial?
}

\author{
Iván López Pardo \\ Universidad Carlos III de Madrid \\ ilpardo@polsoc.uc3m.es
}

Resumen: La existencia de un cambio climático global (CC) está ya constatada en el informe científico del Panel Intergubernamental sobre Cambio Climático (IPCC). Entre las causas de este, se encuentra la actividad bumana junto con las causas naturales del planeta. Todo ello ha dado lugar al desarrollo de diferentes instrumentos de lucha contra el CC, con objetivos que deberán ser alcanzados por las sociedades. Las empresas no son ajenas a este proceso, ni a la causa del problema, ni a su solución. Analizamos en este trabajo cómo encajan la problemática y los objetivos sobre el CC en el contexto de la Responsabilidad Social Empresarial, y los retos a los que se enfrentan las empresas, centrándonos principalmente en el caso de España en el contexto de la Unión Europea.

Palabras clave: Responsabilidad Social Empresarial, cambio climático, empresas, desarrollo sostenible.

\section{Climate Change, A Challenge for Corporate Social Responsibility?}

Abstract: The process of global climate change (CC) bas been confirmed by a scientific report released by the Intergovernmental Panel on Climate Change (IPCC). The reports cites the causes of $\mathrm{CC}$ as being buman activity combined with natural factors. This has led to the development of different instruments to fight CC and the setting of specific goals for societies. Businesses are also implicated in this process, both in terms of the causes of the problem and its solution. In this study we analyse how issues and objectives regarding CC are dealt with in the context of Corporate Social Responsibility. We also investigate the challenges that companies face, focusing primarily on the case of Spain in the context of the European Union.

Keywords: corporate social responsibility, climate change, companies, sustainable development. 
La existencia de un cambio climático planetario (en adelante «CC») es ya algo confirmado por un consenso científico sin fisuras (IPCC, 2013). Recordemos que los tres indicadores del CC son datos empíricos, basados en mediciones: el aumento de la temperatura media de la superficie de la Tierra $\left(0,8{ }^{\circ} \mathrm{C}\right)$, el deshielo de los polos (pérdida de 344.000 millones de toneladas de hielo al año) y el aumento del nivel del mar (1,8 mm/año). El incremento de los episodios meteorológicos extremos (olas de calor, huracanes, inundaciones, entre otros), aunque en rigor no puede ser aplicado al CC en una relación de causa-efecto, es, sin embargo, previsible en los análisis sobre los efectos del CC. El resto de datos, entre ellos el de particular gravedad sobre el posible aumento de la temperatura media entre 2 y $6^{\circ} \mathrm{C}$, son proyecciones basadas en modelos, en probabilidades estadísticas según diversos escenarios futuros.

Las causas del CC son de orden natural pero, en los últimos dos siglos, debido a las actividades humanas desde el inicio de la época industrial, son ante todo de orden social. Así queda reflejado en el informe del Panel Intergubernamental del Cambio Climático (IPCC, 2007 y 2013) con un $95 \%$ de certidumbre. Cabe aclarar que las causas humanas, aunque su apoyatura científica ha ido aumentando, no cuentan con un consenso total ${ }^{1}$ a diferencia del caso de la realidad del CC (IPCC, 2007). Se dan dos posturas contrapuestas: la de los denominados «negacionistas» (corriente científica que niega que el CC sea antropogénico y que, por el contrario, afirma a partir de evidencias empíricas que se ha dado en épocas sucesivas en la evolución del planeta), y la de los defensores de que el calentamiento global está causado por la actividad humana (quienes también demuestran empíricamente su postura y afirman que los negacionistas responden a intereses comerciales de las grandes empresas y no a la veracidad científica).

En cualquier caso, en la época contemporánea, entre las causas sociales del CC destacan la emisión de gases de efecto invernadero (GEI) ${ }^{2}$, debida a la combustión de fósiles y las actividades agrarias, y otras actividades humanas (IPCC, 2013).

El CC ilustra como ningún otro problema social la acuñación sociológica de sociedad del riesgo que caracteriza a las sociedades contemporáneas (Beck, 1992), puesto que es una amenaza que presenta un riesgo civilizatorio, de las condiciones básicas de habitabilidad para las poblaciones humanas. Se plantea así la cuestión sobre cómo abordar el riego climático y los peligros asociados al mismo (aumento del nivel del mar, escasez de agua y olas de calor, entre otros), sobre la adaptación por parte de las comunidades y los individuos y sobre las estrategias que deben

$1<$ http://www.realclimate.org/index.php/archives/2008/10/tropical-tropopshere-iii/>. $2 \mathrm{CO}_{2}, \mathrm{CH}_{4}, \mathrm{~N}_{2} \mathrm{O}, \mathrm{SF}_{6}$, y otros. 
adoptarse (en muchos casos alternativas e innovadoras) y que van dirigidas a una mayor resiliencia social y al debate y la deliberación colectiva necesarios de cara a cambios profundos en las prácticas y en las instituciones (Pelling, 2011: 21).

Por su parte, las consecuencias de dicho CC también son sociales, es decir, afectan a las sociedades e individuos que las componen, bien directamente (por ejemplo, olas de calor o inundaciones ${ }^{3}$ ), bien indirectamente a través de los impactos negativos sobre el medio biofísico (por ejemplo, disminución de la productividad de los suelos) (IPCC, 2007).

Más aún, supone un problema de justicia social o, si se prefiere, de justicia ambiental, dado que las regiones y comunidades socioeconómicamente más desfavorecidas del planeta o de un territorio concreto son las más vulnerables a los efectos del CC, a la vez que son las que menos capacidad de decisión tienen en las instituciones y estructuras de gobernanza mundial que impulsan políticas en el ámbito internacional para afrontar el CC en el conjunto del planeta.

En el ámbito de la política internacional, hace tiempo que se desarrolla un intenso debate en torno a la cuestión de qué supone una «respuesta justa» al cambio climático, así como en torno a la diferencia entre las nociones «distribucionales» y «procedimentales» de la justicia ambiental (Dobson, 1998; Low y Gleeson, 1998; Schlosberg, 2007).

Si bien la mayor parte de los análisis del impacto del CC son de ámbito local (Warren, 2006; Hitz y Smith, 2004), contamos con algunos estudios que aportan modelos de impacto sobre la geografía en el dominio global, como los estudios «Fast Track» (Arnell, 2004; Nicholls, 2004; Arnell et alii, 2002; Levy et alii, 2004; Parry et alii, 2004; Van Lieshout et alii, 2004), con resultados, principalmente, sobre las economías en desarrollo, la disponibilidad de recursos acuíferos $y$, en particular, del agua potable, la seguridad alimentaria, los cambios en los ecosistemas o la exposición a la malaria (Schroeter et alii, 2005). Naciones Unidas llega a resultados similares en su informe Objetivos de Desarrollo del Milenio $(2005)^{4}$, que apela a los elementos comunes entre el desarrollo sostenible y la capacidad de adaptación de las naciones para afrontar el CC y al potencial del CC para determinar el desarrollo de las sociedades.

A partir de los resultados de estos estudios e informes, se puede concluir que el impacto del CC a medio y largo plazo está sujeto no solo a la evolución del CC, sino también a la situación social, económica y tecnológica del mundo; en defini-

3 Toda la zona oeste de Londres se vio afectada, pues Inglaterra ha vivido el invierno más húmedo en casi 250 años. En toda Inglaterra se inundaron unas 6.500 casas.

4 <http://millenniumindicators.un.org/unsd/mi/pdf/MDG\%20BOOK_SP_new.pdf >. Último acceso: $1 / 5 / 2014$. 
tiva, a la organización de las sociedades y a la responsabilidad de la amplia gama de actores sociales presentes en las actividades sociales.

En lo relativo a las políticas sobre el CC, se ha producido en las últimas décadas un cierto consenso. Estas políticas, como veremos a continuación, afectan e implican de lleno a las empresas y, por tanto, al contenido de su política de Responsabilidad Social Empresarial.

Dichas políticas de lucha contra el CC abarcan todos los ámbitos territoriales: el global (Protocolo de Kioto ${ }^{5}$ ), el regional (por ejemplo, Unión Europea ${ }^{6}$ ), el nacional (por ejemplo, Plan Nacional de Mitigación y Adaptación al Cambio Climático de España $a^{7}$, el local (por ejemplo, Red de Ciudades por el Clima ${ }^{8}$ ) y el individual (por ejemplo, certificación energética de las viviendas ${ }^{9}$ ). Asimismo, abarcan todo tipo de políticas (legislativas, económicas, fiscales, sociales...), todo tipo de tecnologías (del automóvil, de la energía, de la alimentación....) y, virtualmente, todo tipo de actividades de la «fábrica social».

Atendiendo a los resultados (IPCC, 2013), es obvia la insuficiencia de dichas políticas, al menos en cuanto a su intensidad y velocidad, mucho menor que la que requeriría una lucha profunda contra el CC (IPCC, 2013); sobre todo, en el objetivo de limitar las emisiones GEI y así controlar la subida de las temperaturas del planeta. Se trata de no traspasar los límites a partir de los cuales la evolución y las consecuencias del CC serían irreversibles.

Es cierto que existe una amplia conciencia de que es preciso hacer más, y más rápido, para combatir el CC. Un referente al respecto es la propuesta de la comisaria de medio ambiente de la UE, Connie Hedegaard, de reducción del 40\% de las emisiones de gases de efecto invernadero con respecto a los datos de 1990.

Sin embargo, al mismo tiempo, la menor competitividad económica que está suponiendo a Europa aplicar estas medidas en relación con otras regiones o potencias económicas mundiales que no las aplican hace que se esté reconsiderando y cuestionando en la actualidad su continuidad, o al menos el alcance de estas medidas. Este es un claro ejemplo de cómo no es posible abordar la lucha contra el CC con garantías sin la coordinación y compromiso internacional, sin un compromiso real de la comunidad internacional en torno a esta problemática.

$5<$ https://unfccc.int/portal_espanol/informacion_basica/protocolo_de_kyoto/items/6215.php >. Último acceso: $11 / 3 / 2014$.

$6<$ http://ec.europa.eu/clima/policies/brief/eu/index_en.htm>. Último acceso: 11/3/2014.

$7<$ http://www.magrama.gob.es/es/cambio-climatico/temas/impactos-vulnerabilidad-y-adaptacion/2_informe_seguimiento_pnacc_tcm7-197096.pdf $>$. Último acceso: 11/3/2014.

$8<$ http://www.redciudadesclima.es/ > Último acceso: 11/3/2014.

$9<$ http://www.minetur.gob.es/energia/desarrollo/eficienciaenergetica/certificacionenergetica/paginas/certificacion.aspx $>$. Último acceso: 11/3/2014. 


\section{Y las empresas, ¿qué están haciendo en relación con el CC?}

La lucha contra el CC es un asunto de tal envergadura que está requiriendo la participación de todos y cada uno de los actores sociales que habitan, trabajan y estudian en las sociedades contemporáneas, bien sea en las económicamente desarrolladas como en las empobrecidas o en vías de desarrollo. Más aún, la lucha contra el CC implica «niveles de cooperación sin precedentes, no solo entre países, sino también entre los distintos niveles gubernamentales y el sector privado» (De Boer, 2009: 1).

Por actores sociales nos referimos, en relación con este tema, no solo a todo el conjunto de instituciones en sentido amplio del término (políticas, económicas, sociales...) y grupos sociales de toda índole, sino también a los individuos particulares con capacidad de acción en su ámbito particular.

No obstante, conviene aclarar que aun existiendo — más que existir realmente, se invoca - esa corresponsabilidad, las responsabilidades de los diferentes actores sociales no son las mismas, ni en cuanto a la causación del problema, ni en cuanto a la capacidad de solución del mismo. Tampoco se trata de una corresponsabilidad sumatoria, descoordinada, sino de una participación en un objetivo común, que coordinada y cooperativamente, multiplique los resultados. Y este es precisamente el déficit mayor, como analizaremos más adelante.

En España, al igual que en el resto de países occidentales, aunque se dan variaciones de año en año, el 55\% de las emisiones de carbono dependen de los sectores denominados difusos (es decir, el transporte, el sector residencial, los hogares, entre otros), mientras que el $45 \%$ son de los sectores fijos (como es el caso de las plantas energéticas e industriales).

La responsabilidad empresarial, en particular de determinadas empresas, es, en cualquier caso, una de las más importantes. Así, en el caso de España, tan solo 10 empresas emiten el 65\% de todas las emisiones de $\mathrm{CO}_{2,}$ y 30 de ellas, el $78 \%$. Hay que diferenciar, por ello, aquellas empresas cuyas actividades forman parte relevante de las causas directas del CC de las que están en otro orden de causación.

Entre las primeras estarían todas las industrias cuyo proceso de producción implica emisiones de gases de efecto invernadero - centrales eléctricas $(51 \%$ de las emisiones), cementeras (19\%), refinerías y petroleras (11\%), siderúrgicas $(8 \%)$, otras industrias $(11 \%)^{10}$. Debido al consumo de combustibles y al uso de materias primas, las emisiones de $\mathrm{C} 02$ de dichas industrias constituyen un de-

10 Plan Nacional de Asignaciones. BOE. Real Decreto 1030/2007, de 20 de julio. 
terminado número de toneladas que forman parte del inventario de emisiones de cada país.

Si bien, por una parte, aunque no es sencillo, es más factible incorporar cambios en las empresas que forman parte de los sectores fijos que en el sector difuso, por el número de actores que implica este último y los cambios de valores y comportamiento que supone para el conjunto de la sociedad; se trata de empresas multinacionales, mercados oligopólicos del sector energético, con gran capacidad de presión sobre los gobiernos y estados, y cuyos centros de decisión a su vez suelen ubicarse fuera de los países donde operan. Una vez más, se ponen de manifiesto las dimensiones y la interdependencia entre lo local y lo global, y las dificultades para la gobernanza que implican, en el caso que nos ocupa, las problemáticas medioambientales de índole planetaria.

A su vez, si bien estas grandes empresas en general están obligadas, en el caso de España y Europa, a formar parte de la política de lucha contra el CC, más concretamente están sujetas al Protocolo de Kioto ${ }^{11}$, los resultados de dicha política, aun con datos positivos, son controvertidos. No es el objeto de este trabajo entrar en detalle en ese análisis, sino poner de manifiesto que ese es uno de los ejes centrales de la nueva situación de las empresas en relación con el nuevo problema del CC.

Además de esa articulación legalmente ${ }^{12}$ obligatoria de las empresas sujetas al Protocolo de Kioto, la integración de la cuestión del CC en las diversas normativas sectoriales ${ }^{13}$ también está implicando a las actividades de empresas de esas y otras características.

Hay que hacer mención también a la cuestión de la gestión ambiental en las empresas, que aun sin estar dirigida directa y exclusivamente hacia el problema del CC, sino hacia la gestión de residuos y demás, tiene alguna conexión con

11 El Protocolo de Kioto, auspiciado por las Naciones Unidas (Convención Marco sobre Cambio Climático), es el acuerdo global de lucha contra el CC, dentro del cual Europa adquirió el compromiso de reducir el 8\% las emisiones de $\mathrm{CO}_{2}$, sobre la base de los niveles existentes en 1990, entre 2008-2013. El compromiso adquirido por España es poder aumentar en un límite máximo del 15\%, utilizando diversos instrumentos: el comercio de emisiones de $\mathrm{CO}_{2}$; y los mecanismos de desarrollo limpio. Actualmente se está negociando el pos-Kioto.

12 En el caso de España, en el marco del Plan Nacional de Adaptación al Cambio Climático. <http://www.magrama.gob.es/es/cambio-climatico/temas/impactos-vulnerabilidad-y-adaptacion/plan-nacional-adaptacioncambio-climatico/default.aspx > (último acceso: 25/3/2014).

13 En España: Ley 33/2011, de 4 de octubre, General de Salud Pública; RD 1274/2011, de 16 de septiembre, Plan Estratégico Estatal del Patrimonio Natural y de la Biodiversidad; Ley 41/2010, de 29 de diciembre, de Protección del Medio Marino; RD 903/2010, de 9 de julio, de Evaluación y Gestión de Riesgos de Inundación; Orden ARM/2656/2008, de 10 de septiembre, por la que se aprueba la Instrucción de Planificación Hidrológica; RD 907/2007, de 6 de julio, por el que se aprueba el Reglamento de la Planificación Hidrológica; Orden ARM/2444/2008, de 12 de agosto, por la que se aprueba el Programa de Acción Nacional de Lucha contra la Desertificación en cumplimiento de la Convención de Naciones Unidas de Lucha contra la Desertificación; Ley 45/2007, de 13 de diciembre, para el Desarrollo Sostenible del Medio Rural. Un segundo nivel de actuación es la incorporación en los procesos de evaluación ambiental (evaluación de impacto ambiental —EIA—y evaluación ambiental estratégica -EAE). 
el problema, aunque sea indirectamente. Nos estamos refiriendo a las diversas certificaciones y herramientas de gestión ambiental que las empresas, en mayor o menor medida, han utilizado (ISO 14.000; UNE 77-801-94; EMAS, entre otras).

Pero, es más, la aparición del CC no solo como fenómeno biogeofísico, sino también como fenómeno social, ha producido multitud de iniciativas empresariales voluntarias al respecto: acuerdos, plataformas ${ }^{14}$, campañas... No hay nada que objetar al respecto, por el contrario, dicha respuesta a una situación sociológica demuestra la relevancia del asunto. Sin embargo, el CC - al igual que ocurre con otros temas de actualidad social - no ha estado exento de lo que se denomina greenwashing o mercadotecnia, que no siempre responde a la actuación real empresarial ${ }^{15}$.

Sin embargo, se ha desarrollado en la última década una herramienta más global que la estrictamente de inventario de emisiones indicada con anterioridad, la denominada buella de carbono. Se trata de una aplicación del análisis del ciclo de vida de productos al caso de las emisiones de gases de efecto invernadero. Conviene recordar aquí que, por ciclo de vida de un producto (de la «cuna» a la «tumba»), se entiende el balance ambiental que evalúa los impactos ambientales de un producto o servicio durante todas y cada una de las etapas de su existencia, desde la extracción de las materias primas, el transporte, la producción y la distribución, hasta el desecho del producto como residuo una vez consumido.

No cabe duda de que es un enfoque que supera el tradicional análisis de costebeneficio empresarial, donde la cuestión medioambiental recibía el nombre de externalidades, manifestando así que era algo ajeno a la empresa.

La huella de carbono puede analizarse para organizaciones en su conjunto, o para productos y servicios concretos.

El análisis de la huella de carbono presenta otra peculiaridad: pone en evidencia cierta corresponsabilidad, como decíamos al principio. En efecto, además de los procesos de producción, que implican de lleno a las empresas correspondientes, el consumo o el uso del producto y la gestión del residuo implican también a los consumidores, puesto que pueden contar con información sobre el producto que les permita tomar una decisión de compra o uso, y además tienen el protagonismo en la correcta separación y depósito del residuo correspondiente.

14 Una de las plataformas internacionales más relevantes es World Business Council for Sustainable Development: <http://www.wbcsd.org/home.aspx> (último acceso: 25/3/2014).

$15 \mathrm{La}$ demanda judicial interpuesta por la Organización de Consumidores (OCU) contra Iberdrola, en 2004, fue sonada. Estaba publicitando que sus consumidores de energía, lo eran de energía verde, cuando no era el caso. Igualmente, en este sentido, y tan solo a modo de mención, en la actualidad las multinacionales de la energía o de las refinerías y petroleras, en el contexto actual de crisis económica, han redirigido sus mensajes e imagen hacia lo social, o la acción social, estrategias sobre las que de nuevo cabe añadir los pertinentes interrogantes. 
Sin embargo, el análisis de la huella de carbono tiene ciertas dificultades. Su cálculo puede ser objeto de incertidumbre debido a que la información que se requiere para cumplimentar todas y cada una de las fases indicadas puede llegar a ser muy amplia, e incluso pueden no existir esos análisis; por eso, se suele tender a utilizar datos secundarios o a hacer metaanálisis, lo que obviamente puede suscitar controversia sobre su rigor.

A pesar de ello, el éxito de esta herramienta se pone de manifiesto en la reciente noticia (referencia) de que las 50 mayores empresas españolas apuestan por calcular su huella de carbono. Y, es más, la gran mayoría de las firmas españolas calculan su huella de carbono e incluyen en sus informes un capítulo sobre CC. Además, informan sobre sus objetivos de reducción de emisiones de gases de efecto invernadero y del consumo energético. La mayoría calcula su huella de carbono no solo teniendo en cuenta el alcance de las emisiones directas, sino también el de las emisiones indirectas. Y un alto porcentaje de empresas reflejan las acciones destinadas a reducir su impacto ambiental. Además, se da una correlación entre las empresas que han implantado un Sistema de Gestión Ambiental y el cálculo de la huella de carbono, y tienen mayor participación en la organización internacional Carbon Disclosure Project (CDP), proyecto dedicado al control de las emisiones de carbono de las empresas.

Otra corresponsabilidad que ha permitido el análisis de huella de carbono es la denominada neutralización de las emisiones. Se trata, en este caso, de compensar y contrarrestar, bien en el caso de empresas, bien en el caso de particulares, las emisiones de gases de efecto invernadero que se producen por una actividad concreta y que no pueden ser eliminadas al momento con la tecnología actual, con otra actividad que reduzca la misma cantidad, en un punto u otro del planeta, indistintamente. Este enfoque ilustra la oferta que hacen algunas compañías aéreas para que los pasajeros de sus vuelos paguen una cantidad de dinero extra para compensar su huella de carbono en ese viaje con otra actividad que la neutralice (un proyecto de forestación, por ejemplo).

Es obvio que son medidas, de nuevo, con potencialidad de suscitar controversia de muchos órdenes, desde el moral (el impacto ambiental del vuelo no se elimina, sino que se mitiga por otro lado), hasta el de tender a poner la «culpabilidad», y, por tanto, la penitencia, en el punto final, en el usuario. Dejando claros los distintos niveles de responsabilidad en las corresponsabilidades, como decíamos, no cabe duda de que en cualquier caso un individuo particular tiene también en su mano la decisión de utilizar o no uno de los medios (transporte aéreo) que es una de las causas más directas del CC. 
Las diferencias culturales se presentan también como relevantes en cuanto a la adjudicación de responsabilidades del CC. Es ilustrativo el resultado del Eurobarómetro sobre el cambio climático (EU, 2011), en el resultado de la pregunta sobre a quién atribuyen los entrevistados la responsabilidad de abordar el problema del CC, los suecos destacan (45\%) por atribuirse a ellos mismos personalmente dicha responsabilidad, y los españoles, al Gobierno (57\%).

\section{¿Cómo encaja e interactúa la lucha contra el CC en el marco de la Responsabilidad Social Empresarial?}

El problema del CC, con su relevancia social, política y económica, ha irrumpido de tal manera en el ámbito empresarial, que, necesariamente, ha planteado su relación con la Responsabilidad Social Empresarial (RSE).

Otros trabajos de este número monográfico de la revista han profundizado en la materia de la Responsabilidad Social Empresarial. Aquí, por tanto, nos limitamos a unas mínimas referencias que permitan una autonomía en este análisis sobre su relación con el CC.

Conviene tener en cuenta que durante las últimas décadas la RSE ha ido creciendo desde una noción estrecha y marginalizada hacia un concepto complejo y multifacético que se va convirtiendo actualmente en una cuestión central en la toma de decisiones corporativas (Cochran, 2007), principalmente en Europa y Norteamérica. Dicha evolución se sintetiza de la siguiente manera: 1) de la filantropía a la filantropía estratégica; 2) de la inversión a la inversión socialmente responsable; 3) del negocio al negocio social; 4) de los fondos de capital ventura a los fondos de capital ventura social; 5) de los másteres en business administration (MBA) a los MBA en RSE.

No es el objeto de este artículo analizar esas afirmaciones, pero es de utilidad para entender el camino que ha hecho posible la incorporación de las políticas contra el CC en la RSE.

Así, en lo que al asunto de este artículo respecta, cabe reseñar que el movimiento hacia una sociedad sostenible, que parte del informe Nuestro Futuro Común (ONU, 1987), ha evolucionado de una manera imparable, en especial, desde la Cumbre de la Tierra en Río de Janeiro en 1992. El CC se incorpora al conjunto de conflictos que se deben abordar en el equilibrio necesario entre las dimensiones económica, ambiental y social de la sostenibilidad planetaria, y empiezan a surgir los principales instrumentos en torno a los que se está articulando 
mundialmente la idea de la sostenibilidad corporativa ${ }^{16}$; destacan por su relevancia los índices de sostenibilidad Dow Jones Sustainability Index y el FTSE4Good.

Es destacable en el marco europeo la reciente aprobación del Parlamento Europeo de la Directiva ${ }^{17}$ sobre divulgación de información no financiera, que obligará (una vez ratificada por el Consejo) a unas 6.000 grandes empresas de la Unión Europea (entre 500 y 600 españolas) a informar sobre sus impactos en materia social, ambiental, políticas de diversidad, respeto de los derechos humanos y medidas contra la corrupción y el soborno.

Las propias empresas han mostrado una evolución en su perspectiva sobre el CC, desde los primeros enfoques de tipo reactivo, intentando influir en el debate político, a las estrategias corporativas que cada vez más incluyen respuestas económicas a la cuestión del CC, como pueden ser cambios tecnológicos, mayor eficiencia en los procesos de producción y en los propios productos, mecanismos de compensación económica, entre otros.

Es decir, las empresas se enfrentan a la imperiosa necesidad que les plantea el CC de evolucionar desde el business as usual (o más de lo mismo, o el path dependency), a una nuevo cultura empresarial que aproveche las ventanas de las oportunidades que ofrece el CC (cambios económicos, tecnológicos y sociales) en un mundo globalizado y líquido (Bauman, 1999).

Sin embargo, la capacidad del marco de la Responsabilidad Social Empresarial para incluir la cuestión del desarrollo sostenible y del CC tiene limitaciones. Aun situando la importancia de una plena inclusión del problema por parte de las políticas de RSE de las empresas, es justo reconocer que la solución de la crisis del CC excede los límites de la RSE de las empresas. En esa línea, Toms (2014) plantea que los directivos de las empresas no tienen tanto poder como las fuerzas del mercado actual, que se dirigen a un continuo aumento del consumo de los recursos del mundo. Para este autor, la clave es, en particular, la fuerte asociación entre el desarrollo de los recursos del petróleo, los mercados mundiales y la capacidad productiva, siendo esos los determinantes centrales del CC.

Así, si los estados pretenden un claro compromiso con la solución del CC (Prieto, 2014), deberán enviar una señal clara a las empresas, con el fin de tender

16 Global Reporting Iniciative (GRI), (1999); la Norma Marco AA1000 (1999); los índices de sostenibilidad Dow Jones Sustainability Index (1999); la International Standard on Assurance Engagementes ISAE 100 (2000); las directrices de la OCDE para las empresas multinacionales (2000); el Libro Verde para fomentar un marco europeo para la Responsabilidad Social de las Empresas de la Unión Europea (2001); la Declaración tripartita de principios sobre las empresas multinacionales y la política social de la OIT (2002); la ISO 26.000 (2010); la Estrategia renovada de la UE para 2011-2014 sobre RSE (2011).

17 P7_TA-PROV(2014)0368 Disclosure of non-financial and diversity information by certain large companies and groups ${ }^{* * *}$ European Parliament legislative resolution of 15 April 2014 on the proposal for a directive of the European Parliament and of the Council amending Council Directives 78/660/EEC and 83/349/ $\mathrm{EEC}$ as regards disclosure of non-financial and diversity information by certain large companies and groups (COM(2013)0207 -C7-0103/2013 -2013/0110(COD). 
hacia una economía baja en carbono, que a medio y a largo plazo será la economía competitiva, innovadora y sostenible.

Para el caso de España, Prieto (2014) insiste en que si se pretende avanzar en la senda de una economía baja en carbono, las empresas tienen que disminuir sus emisiones de CO2, y el Gobierno debe cambiar la matriz energética, actualmente basada en el carbón y en los combustibles fósiles. A la vez, deben potenciarse las energías renovables que no emiten $\mathrm{CO} 2$. También debe reforzarse el transporte público y, especialmente, el tren de mercancías, con el fin de reducir así el transporte privado y las emisiones de petroleras y refinerías. Además, esto disminuiría la elevadísima dependencia energética y también la muy importante salida de divisas de este país.

\section{La transición hacia las sociedades neutras en carbono y la responsabilidad empresarial}

La transición hacia las sociedades descarbonizadas, neutras en carbono o con bajas emisiones en carbono, implica necesariamente, aunque no exclusivamente, la transición de las economías energéticas actuales en la forma y modos en que la energía se genera, se distribuye, se provee y se consume. Como se ha podido observar en el presente artículo, el sector energético y el grupo reducido de empresas que en él operan son una de las causas más destacadas del CC y, por tanto, también es en este espacio en el que cabe articular buena parte de las soluciones.

Así, de cara a alcanzar un futuro económico, ambiental y social sostenible para las generaciones presentes y las venideras, se presenta como un reto para las grandes compañías energéticas abordar un cambio de mentalidad y cultura empresarial que implique nuevas prácticas dirigidas a un horizonte en el que la energía sea más accesible (estamos hablando de los precios de la energía, y así también de la denominada pobreza energética que en la actualidad se estima que afecta al $10 \%$ de los hogares) ${ }^{18}$, más limpia (invertir en energías renovables) y un modelo descentralizado a la vez que regulado que posibilite la presencia de múltiples operadores o la generación autónoma y local de energía.

Negar el CC y sus consecuencias implica abstenerse de asumir la irreversibilidad del mismo, hipotecar el futuro social, y así también el desarrollo empresarial, y proseguir hacia un punto de no retorno de consecuencias imprevisibles.

Estamos hablando, en consecuencia, de una visión integra e integral, holística y a largo plazo, hacia un desarrollo sostenible, donde el CC y el necesario cambio de cultura y mentalidad empresarial en la lucha contra el mismo implica vislum-

18 Observatorio de la Sostenibilidad (2012): Sostenibilidad en España 2012. Capítulo especial energía sostenible para todos. 
brarlo no como una barrera para el desarrollo empresarial, sino por el contrario como una «ventana de oportunidades» a partir del impulso de un debate integrador del conjunto de la sociedad y los actores sociales.

Las medidas hasta ahora presentes en la lucha contra el CC, explicadas anteriormente, y en particular las que conciernen a las empresas (ya sean energéticas o de otros sectores, grandes empresas o medianas y pequeñas), ha quedado probado que son insuficientes, y esto no carece de importancia, dado que supone perder un tiempo muy valioso ante el vertiginoso avance del CC y sus cada vez más notables consecuencias. Se trata de políticas «blandas» o de una transición «débil» hacia las sociedades sostenibles. Es decir, que no suponen un giro sustancial, amplio y profundo hacia las sociedades bajas en carbono, sino que por el contrario se trata de cambios superficiales y tímidos, poco decididos, que en un horizonte no muy lejano limitan de manera irreversible la propia estabilidad de los proyectos empresariales.

Por otra parte, como decíamos, el ámbito de actuación no se restringe únicamente al del sector energético o al de las grandes empresas, sino que implica igualmente a las fuentes no fijas o a sectores difusos en todas las actividades asociadas a los distintos sectores de producción, servicios y consumo. Primar el transporte público sobre el privado, los automóviles basados en energías limpias, la moderación de la construcción basada en criterios de eficiencia energética y de sociabilidad, la rehabilitación energética de los hogares, que supondría una oportunidad empresarial y de generación de empleo (más de la mitad de los edificios españoles tienen más de 30 años de antigüedad, y el 50\% de estos tienen más de 50 años), entre muchos otros, son algunas de las líneas de acción que implican no ya a la clase política, sino a las empresas presentes en estas actividades.

En la transición hacia las sociedades sostenibles y, en particular, hacia las neutras en carbono, la tecnología, el conocimiento científico o la innovación y experimentación, aunque imprescindibles, no son suficientes por sí mismos y tampoco son el principal eje vertebrador de la responsabilidad empresarial. Se trata de un proceso cuya eficacia a medio y largo plazo necesita de la concienciación colectiva, de la legitimación social y, por tanto, de la implementación adecuada, a partir de un saber hacer especializado, de una tecnología social, de procesos de deliberación y participación pública inclusivos que involucren a actores sociales en todos los niveles de los procesos productivos y de consumo.

La aceptación de esta idea, de esta forma de abordar los conflictos y problemáticas en torno a los bienes comunes, como es el medioambiente y la salud del planeta, implica un cambio más que sustantivo en los discursos sociales y el imaginario colectivo y, por tanto, igualmente en la cultura empresarial. 
A su vez, la negociación y el acuerdo colectivos implican dinámicas complejas, entre otras razones, por la presencia de intereses contrapuestos entre actores sociales, y en las que la puesta en marcha frustrada o el incumplimiento de las expectativas creadas hace difícil la recuperación de la confianza en este tipo de procesos.

En definitiva, estamos hablando de la necesidad o, más aún, de la inevitabilidad de un «pacto social» en torno al CC, resultado de la participación del conjunto de la sociedad y de la implicación y compromiso especial del sector empresarial, todo ello entendido como un proceso inevitable.

\section{Conclusiones}

El Cambio Climático está suponiendo un reto de gran envergadura para las empresas, tanto por los profundos cambios que la lucha contra el CC requiere, como por la necesaria velocidad de los mismos. El reto es de orden económico y tecnológico, pero también cultural, de asumir realmente la corresponsabilidad que las empresas tienen tanto en la causa del problema como en su solución.

El instrumento de la Responsabilidad Sociedad Empresarial se muestra como un marco relevante para abordar dicho reto. Sin embargo, la amplia experiencia empresarial en el pilar medioambiental de la RSE no había incluido tradicionalmente la cuestión del CC, como asunto relativamente nuevo que ha tomado una dimensiones amenazantes para las sociedades.

La lucha contra el CC en el ámbito empresarial es un asunto que requiere todavía de una construcción de capacidad al respecto en el seno de las empresas, y concretamente en el marco de la RSE, que no ha hecho más que empezar, pero que, dadas las características del problema, tendrá un largo recorrido y conllevará décadas, si no siglos, de cambios en el funcionamiento de las sociedades y, lógicamente, de las empresas.

Con independencia de la centralidad de la responsabilidad de los gobiernos en lo relativo al desarrollo de marcos legislativos, económicos, sociales, entre otros, que faciliten dichos cambios sociales, las empresas, a través de la RSE, asumen su parte en la corresponsabilidad de dicho cambio social.

En definitiva, estamos hablando de la necesidad de un «pacto social» sobre la amenaza del CC, que implique al conjunto de la sociedad con el compromiso especial del sector empresarial. 


\section{Referencias}

Arnell, N.W. (2004). «Climate change and global water resources: SRES emissions and socio-economic scenarios». Global Environmental Change, 14: $31-52$.

Arnell, N.W.; M.G.R. Cannell; M. Hulme; R.S. Kovats; J.F.B. Mitchell; R.J. Nicholls; M.L. Parry; M.T.J. Livermore et alii (2002). «The consequences of $\mathrm{CO} 2$ stabilization for the impacts of climate change». Climatic Change, 53: 413-446.

Bauman, Z. (1999). Modernidad líquida. Buenos Aires: Fondo de Cultura Económica.

BeCK, Ulrich (1992), Risk Society: Towards a New Modernity. London: Sage.

Cochran, Philip L. (2007). «The evolution of corporate social responsibility». Business Horizons, 50: 449-454.

De Boer, Y. (2009). Keynote Speech, Sustainable Development in Times of Crises - United Nations Framework Convention on Climate Change.

EU (2011). Climate Change. Special Eurobarometer 372. Accesible en: <http:// ec.europa.eu/public_opinion/archives/ebs/ebs_372_en.pdf >.

Hitz, S. y Sмiтн, J. (2004). «Estimating global impacts from climate change». Global Environmental Change, 14: 201-218.

Leão, Susana y Bala, Alba (2014). Tendencias de reporting sobre la buella de carbono de las 50 mayores empresas españolas. Escola Superior de Comerç Internacional-Universitat Pompeu Fabra. Cátedra MANGO de Responsabilidad Social Corporativa. Documento de trabajo n. ${ }^{\circ} 18$.

Nicholls, R.J. (2004). «Coastal flooding and wetland loss in the 21st century: changes under the SRES climate and socio-economic scenarios». Global Environmental Change, 14:69-86.

Parry, M.L.; C. Rosenzweig; A. Iglesias; M. Livermore y G. Fischer (2004). «Effects of climate change on global food production under SRES emissions and socio-economic scenarios». Global Environmental Change, 14: $53-67$.

Pelling, M. (2011). Adaptation to Climate Change: from resilience to transformation. London: $\mathrm{T}$ and $\mathrm{F}$ Books.

Prieto, F. (2014). «Diez empresas emiten el 65\% del $\mathrm{CO}_{2}$ procedente de sectores industriales y energéticos de España». Público, 4/12/2013. Accesible en: $<$ https://www.diagonalperiodico,net/global/20984-top-ten-empresas-mascontaminan.html>. 
Schroeter, D.; W. Cramer; R. Leemans; I.C. Prentice; M.B. Araujo; N.W. Arnell; A. Bondeau; H. Bugmann (2005). «Ecosystem service supply and vulnerability to global change in Europe». Science, 310: 1333-1337.

Toms, S. (2014). «Economic development, climate change and the limitations of corporate social responsibility». En Kathryn Haynes, Alan Murray, Jesse Dillard (eds.), Corporate Social Responsibility, A Research Handbook, Abingdon: Routledge.

Van Lieshout, M.; R.S. Kovats; M.T.J. Livermore y P. Martens (2004). "Climate change and malaria: analysis of the SRES climate and socioeconomic scenarios». Global Environmental Change, 14: 87-99.

Warren, R. (2006). «Spotlighting impacts functions in integrated assessment models». Working Paper 91. Norwich: Tyndall Centre for Climate Change Research. 